\title{
International publications of authors from Bosnia and Herzegovina in Current Contents indexed publications in the second half of $2015^{\star}$
}

Ačkar Đ, Babić J, Jozinović A, Miličević B, Jokić S, Miličević R, Rajič M, Šubarić D. Starch Modification by Organic Acids and Their Derivatives: A Review. Molecules. 2015 Oct 27;20(10):19554-70. doi: 10.3390/molecules201019554.

Barišić T, Šutalo N, Letica L, Kordić AV. Rupture of splenic artery aneurysm in primipara five days after cesarean section: case report and review of the literature. Wien Klin Wochenschr. 2015 Nov;127(2122):896-8. doi: 10.1007/s00508-015-0856-x. Epub 2015 Sep 15.

Bergsland J, Mirtaheri P, Hiorth N, Fosse E. Review of the endovascular approach to mitral valve disease. Minim Invasive Ther Allied Technol. 2015;24(5):2828. doi: 10.3109/13645706.2015.1065891. Epub 2015 Jul 23.

Bjelanović V, Babić D, Hodžić D, Bjelanović A, Krešić T, Dugandžić-Šimić A, Orešković S. Correlation of psychological symptoms with cortisol and CRP levels in pregnant women with metabolic syndrome. Psychiatr Danub. 2015 Dec;27 Suppl 2:578-85.

Bugiardini R, Cenko E, Ricci B, Vasiljevic Z, Dorobantu M, Kedev S, Vavlukis M, Kalpak O, Puddu PE, Gustiene O, Trninic D, Knežević B, Miličić D, Gale CP, Manfrini O, Koller A, Badimon L. Comparison of Early Versus Delayed Oral $\beta$ Blockers in Acute Coronary Syndromes and Effect on Outcomes. Am J Cardiol. 2016 Mar 1;117(5):760-7. doi: 10.1016/j.amjcard.2015.11.059. Epub 2015 Dec 13.

Bukvić N, Marinović M, Bakota B, Veršić AB, Karlo R, Kvesić A, Gusić N, Čoklo M, Bukvić F. Complications of ESIN osteosynthesis--Experience in 270 patients. Injury. 2015 Nov;46 Suppl 6:S40-3. doi: 10.1016/j.injury.2015.10.042. Epub 2015 Nov 10.

Campos CM, Stanetic BM, Farooq V, Walsh S, Ishibashi Y, Onuma Y, Garcia-Garcia HM, Escaned
J, Banning A, Serruys PW; SYNTAX II Study Group. Risk stratification in 3-vessel coronary artery disease: Applying the SYNTAX Score II in the Heart Team Discussion of the SYNTAX II trial. Catheter Cardiovasc Interv. 2015 Nov 15;86(6):E229-38. doi: 10.1002/ccd.25907. Epub 2015 May 6.

Catic T. Access to Biological Therapies and Consumption Trends in Bosnia and Herzegovina. Value Health. 2015 Nov;18(7):A655. doi: 10.1016/j. jval.2015.09.2364. Epub 2015 Oct 20.

Catic T. Differences In Reimbursement Prices And Inequalities To Access Most Commonly Prescribed Medicines In Bosnia And Herzegovina. Value Health. 2015 Nov;18(7):A527. doi: 10.1016/j. jval.2015.09.1630. Epub 2015 Oct 20.

Choe JY, Prodanovic N, Niebrzydowski J, Staykov I, Dokoupilova E, Baranauskaite A, Yatsyshyn R, Mekic M, Porawska W, Ciferska H, JedrychowiczRosiak K, Zielinska A, Choi J, Rho YH, Smolen JS. A randomised, double-blind, phase III study comparing SB2, an infliximab biosimilar, to the infliximab reference product Remicade in patients with moderate to severe rheumatoid arthritis despite methotrexate therapy. Ann Rheum Dis. 2015 Aug 28. pii: annrheumdis-2015-207764. doi: 10.1136/ annrheumdis-2015-207764. [Epub ahead of print]

Ćurguz Z, Stojanovska Z, Žunić ZS, Kolarž P, Ischikawa T, Omori Y, Mishra R, Sapra BK, Vaupotič J, Ujić P, Bossew P. Long-term measurements of radon, thoron and their airborne progeny in 25 schools in Republic of Srpska. J Environ Radioact. 2015 Oct;148:163-9. doi: 10.1016/j. jenvrad.2015.06.026. Epub 2015 Jul 12.

Damjanov I, Vranic S, Skenderi F. Does everything a surgeon takes out have to be seen by a pathologist? A review of the current pathology practice. Virchows Arch. 2016 Jan;468(1):69-74. doi: 10.1007/s00428015-1801-0. Epub 2015 Jul 9.

${ }^{\star}$ Data for this survey were collected from PubMed/MEDLINE using the keywords Bosnia and Herzegovina and 2015. 
Damjanovska M, Cvetko E, Hadzic A, Seliskar A, Plavec T, Mis K, Vuckovic Hasanbegovic I, Stopar Pintaric T. Neurotoxicity of perineural vs intraneuralextrafascicular injection of liposomal bupivacaine in the porcine model of sciatic nerve block. Anaesthesia. 2015 Dec;70(12):1418-26. doi: 10.1111/anae.13189. Epub 2015 Sep 4.

Debeljak M, Toplak N, Abazi N, Szabados B, Mulaosmanović V, Radović J, Perko D, Vojnović J, Constantin T, Kuzmanovska D, Avčin T. The carrier rate and spectrum of MEFV gene mutations in central and southeastern European populations. Clin Exp Rheumatol. 2015 Nov-Dec;33(6 Suppl 94):S1923. Epub 2015 Sep 24.

Denislic M, Tiric-Campara M, Resić H, Al-Hashel JY, Zorec R, Gojak R, Ravnik J. A neurophysiological study of large- and small-diameter nerve fibers in the hands of hemodialysis patients. Int Urol Nephrol. 2015 Nov;47(11):1879-87. doi: 10.1007/s11255-0151117-7. Epub 2015 Oct 3.

Dujic T, Causevic A, Bego T, Malenica M, VelijaAsimi Z, Pearson ER, Semiz S. Organic cation transporter 1 variants and gastrointestinal side effects of metformin in patients with Type 2 diabetes. Diabet Med. 2016 Apr;33(4):511-4. doi: 10.1111/dme.13040. Epub 2015 Dec 24.

Džoić Dominković M, Ivanac G, Kelava T, Brkljačić B. Elastographic features of triple negative breast cancers. Eur Radiol. 2016 Apr;26(4):1090-7. doi: 10.1007/s00330-015-3925-7. Epub 2015 Aug 1.

Fan T, Grychtol P, Knut R, Hernández-García C, Hickstein DD, Zusin D, Gentry C, Dollar FJ, Mancuso CA, Hogle CW, Kfir O, Legut D, Carva K, Ellis JL, Dorney KM, Chen C, Shpyrko OG, Fullerton EE, Cohen O, Oppeneer PM, Milošević DB, Becker A, Jaroń-Becker AA, Popmintchev T, Murnane MM, Kapteyn HC. Bright circularly polarized soft $\mathrm{X}$-ray high harmonics for X-ray magnetic circular dichroism. Proc Natl Acad Sci U S A. 2015 Nov 17;112(46):14206-11. doi: 10.1073/pnas.1519666112. Epub 2015 Nov 3.

Gyberg V, De Bacquer D, De Backer G, Jennings C, Kotseva K, Mellbin L, Schnell O, Tuomilehto J, Wood D, Rydén L, Amouyel P, Bruthans J, Conde AC, Cifkova R, Deckers JW, De Sutter J, Dilic M, Dolzhenko M, Erglis A, Fras Z, Gaita D, Gotcheva $\mathrm{N}$, Goudevenos J, Heuschmann P, Laucevicius A, Lehto S, Lovic D, Miličić D, Moore D, Nicolaides E, Oganov R, Pająk A, Pogosova N, Reiner Z, Stagmo M, Störk S, Tokgözoğlu L, Vulic D; EUROASPIRE Investigators. Patients with coronary artery disease and diabetes need improved management: a report from the EUROASPIRE IV survey: a registry from the EuroObservational Research Programme of the European Society of Cardiology. Cardiovasc Diabetol. 2015 Oct 1;14:133. doi: 10.1186/s12933-015-0296-y.

Hadziabdic N, Kurtovic-Kozaric A, Pojskic N, Sulejmanagic N, Todorovic L. Gene-expression analysis of matrix metalloproteinases 1 and 2 and their tissue inhibitors in chronic periapical inflammatory lesions. J Oral Pathol Med. 2016 Mar;45(3):224-30. doi: 10.1111/jop.12347. Epub 2015 Aug 21.

Hadžimehmedagić A, Gavrankapetanović I, Vranić $\mathrm{H}$, Sunje M, Gavrankapetanović F, Papović A, Granov N, Begović B, Omerović Đ. Haemodynamic assessment in simulated operative positions before knee arthroplasty can be useful in deep vein thrombosis prediction. Int Orthop. Hodzic E, Rasic S, Klein C, Covic A, Unsal A, Cunquero JM, Prischl FC, Gauly A, Kalicki RM, Uehlinger DE. Clinical Validation of a Peritoneal Dialysis Prescription Model in the PatientOnLine Software. Artif Organs. 2016 Feb;40(2):144-52. doi: 10.1111/aor.12526. Epub 2015 Jul 6.

Hagl M, Powell S, Rosner R, Butollo W. Dialogical Exposure with Traumatically Bereaved Bosnian Women: Findings from a Controlled Trial. Clin Psychol Psychother. 2015 Nov;22(6):604-18. doi: 10.1002/cpp.1921. Epub 2014 Sep 24.

Hermans LE, Svicher V, Pas SD, Salpini R, Alvarez M, Ben Ari Z, Boland G, Bruzzone B, Coppola N, Seguin-Devaux C, Dyda T, Garcia F, Kaiser R, Köse S, Krarup H, Lazarevic I, Lunar MM, Maylin S, Micheli V, Mor O, Paraschiv S, Paraskevis D, Poljak M, Puchhammer-Stöckl E, Simon F, Stanojevic M, Stene-Johansen K, Tihic N, Trimoulet P, Verheyen J, Vince A, Weis N, Yalcinkaya T, Lepej SZ, Perno C, Boucher CA, Wensing AM; HEPVIR Working Group of the European Society for Translational Antiviral Research. Combined Analysis of the Prevalence of Drug-Resistant Hepatitis B Virus in Antiviral Therapy-Experienced Patients in Europe (CAPRE). J Infect Dis. 2016 Jan 1;213(1):39-48. doi: 10.1093/ infdis/jiv363. Epub 2015 Jul 1.

Hodzic E, Rasic S, Klein C, Covic A, Unsal A, Cunquero JM, Prischl FC, Gauly A, Kalicki RM, Uehlinger DE. Clinical Validation of a Peritoneal Dialysis Prescription Model in the PatientOnLine Software. Artif Organs. 2016 Feb;40(2):144-52. doi: 10.1111/aor.12526. Epub 2015 Jul 6.

Jakovljević M, Ostojić L. Person-centered medicine and good clinical practice: disease has to be cured, but the patient has to be healed. Psychiatr Danub. 2015 Dec;27 Suppl 2:546-9. 
Janković J, Marinković J, Stojisavljević D, Erić M, Vasiljević N, Janković S. Sex inequalities in cardiovascular health: a cross-sectional study. Eur J Public Health. 2016 Feb;26(1):152-8. doi: 10.1093/ eurpub/ckv161. Epub 2015 Sep 14.

Kajić M, Tomić V, Martinac M, Mikulić-Kajić M, Orlović M. Medical students' attitudes towards contraception. Psychiatr Danub. 2015 Dec;27 Suppl 2:590-2.

Kasum M, von Wolff M, Franulić D, Čehić E, Klepac-Pulanić T, Orešković S, Juras J. Fertility preservation options in breast cancer patients. Gynecol Endocrinol. 2015;31(11):846-51. doi: 10.3109/09513590.2015.1081684. Epub 2015 Sep 15.

Kiss O, Tőkés AM, Vranic S, Gatalica Z, Vass L, Udvarhelyi N, Szász AM, Kulka J. Expression of miRNAs in adenoid cystic carcinomas of the breast and salivary glands. Virchows Arch. 2015 Nov;467(5):551-62. doi: 10.1007/s00428-015-1827-3. Epub 2015 Aug 21.

Korda-Vidić V, Vasilj I, Babić D. The stress of war and breast cancer incidence. Psychiatr Danub. 2015 Dec;27 Suppl 2:571-7.

Kučinić M, Previšić A, Graf W, Mihoci I, Šoufek M, Stanić-Koštroman S, Lelo S, Vitecek S, Waringer J. Larval description of Drusus bosnicus Klapálek 1899 (Trichoptera: Limnephilidae), with distributional, molecular and ecological features. Zootaxa. 2015 May 13;3957(1):85-97. doi: 10.11646/zootaxa.3957.1.7.

Kujundzic B, Zeljic K, Supic G, Magic M, Stanimirovic D, Ilic V, Jovanovic B, Magic Z. Association of vdr, cyp27b1, cyp24a1 and mthfr gene polymorphisms with oral lichen planus risk. Clin Oral Investig. 2016 May;20(4):781-9. doi: 10.1007/ s00784-015-1572-7. Epub 2015 Aug 26.

Kurtovic-Kozaric A, Hasic A, Radich JP, Bijedic V, Nefic H, Eminovic I, Kurtovic S, Colakovic F, Kozaric M, Vranic S, Bovan NS. The reality of cancer treatment in a developing country: the effects of delayed TKI treatment on survival, cytogenetic and molecular responses in chronic myeloid leukaemia patients. Br J Haematol. 2016 Feb;172(3):420-7. doi: 10.1111/bjh.13843. Epub 2015 Nov 11.

Lipovac K, Tešić M, Marić B, Đerić M. Self-reported and observed seat belt use--A case study: Bosnia and Herzegovina. Accid Anal Prev. 2015 Nov;84:74-82. doi: 10.1016/j.aap.2015.08.010. Epub 2015 Aug 31.

Lukenda A, Dotlic S, Vukojevic N, Saric B, Vranic $\mathrm{S}$, Zarkovic K. Expression and prognostic value of putative cancer stem cell markers CD117 and CD15 in choroidal and ciliary body melanoma. J Clin Pathol. 2016 Mar;69(3):234-9. doi: 10.1136/ jclinpath-2015-203130. Epub 2015 Aug 19.

Mahmić-Kaknjo M, Kadić D, Hodžić H, SpahićSarajlić S, Hadžić E, Ademović E. Awareness, knowledge, use, and attitudes toward evidence based medicine in a developing country: survey of physicians in a canton in Bosnia and Herzegovina. Croat Med J. 2015 Dec;56(6):558-66.

Marković-Peković V, Škrbić R, Petrović A, VlahovićPalčevski V, Mrak J, Bennie M, Fadare J, Kwon HY, Schiffers K, Truter I, Godman B. Polypharmacy among the elderly in the Republic of Srpska: extent and implications for the future. Expert Rev Pharmacoecon Outcomes Res. 2015 Dec 15:1-10. [Epub ahead of print]

Meisrimler CN, Menckhoff L, Kukavica BM, Lüthje S. Pre-fractionation strategies to resolve pea (Pisum sativum) sub-proteomes. Front Plant Sci. 2015 Oct 19;6:849. doi: 10.3389/fpls.2015.00849. eCollection 2015.

Milicevic R, Jaksic N, Aukst-Margetic B, Jakovljevic M. Personality traits and treatment compliance in patients with type 2 diabetes mellitus. Psychiatr Danub. 2015 Dec;27 Suppl 2:586-9.

Mirjanic-Azaric B, Stojakovic-Jelisavac T, Vukovic B, Stojanovic D, Vujnic M, Uletilovic S. The impact of time of sample collection on the measurement of thyroid stimulating hormone values in the serum. Clin Biochem. 2015 Dec;48(18):1347-9. doi: 10.1016/j.clinbiochem.2015.08.020. Epub 2015 Aug 20.

Mišigoj-Duraković M, Sorić M, Matika D, Jukić I, Duraković Z. Which is more important for reducing the odds of metabolic syndrome in men: Cardiorespiratory or muscular fitness? Obesity (Silver Spring). 2016 Jan;24(1):238-44. doi: 10.1002/ oby.21264. Epub 2015 Dec 6.

Namy S, Heilman B, Stich S, Crownover J, Leka B, Edmeades J. Changing what it means to 'become a man': participants' reflections on a school-based programme to redefine masculinity in the Balkans. Cult Health Sex. 2015;17 Suppl 2:206-22. doi: $10.1080 / 13691058.2015 .1070434$.

Novokmet N, Galov A, Marjanović D, Škaro V, Projić P, Lauc G, Primorac D, Rudan P. Genetic structure and admixture between Bayash Roma from northwestern Croatia and general Croatian population: evidence from Bayesian clustering 
analysis. Anthropol Anz. 2015;72(3):321-34. doi: 10.1127/anthranz/2015/0485. Epub 2015 Aug 3.

Obradovic B, Obradovic M. Triple V-Y Vermilion Augmentation of the Upper Lip. J Craniofac Surg. 2015 Nov;26(8):e736-8. doi: 10.1097/ SCS.0000000000002246.

Petric I, Mustafić N. Dynamic modeling the composting process of the mixture of poultry manure and wheat straw. J Environ Manage. 2015 Sep 15;161:392-401. doi: 10.1016/j.jenvman.2015.07.033. Epub 2015 Jul 24.

Pilav A, Rudić A, Branković S, Djido V. Perception of health risks among adolescents due to consumption of cigarettes, alcohol and psychoactive substances in the Federation of Bosnia and Herzegovina. Public Health. 2015 Jul;129(7):963-9. doi: 10.1016/j. puhe.2015.05.004. Epub 2015 Aug 6.

Pojskic L, Haveric S, Lojo-Kadric N, Hadzic M, Haveric A, Galic Z, Galic B, Vullo D, Supuran CT, Milos M. Effects of dipotassium-trioxohydroxytet rafluorotriborate, $\mathrm{K}(2)[\mathrm{B}(3) \mathrm{O}(3) \mathrm{F}(4) \mathrm{OH}]$, on cell viability and gene expression of common human cancer drug targets in a melanoma cell line. J Enzyme Inhib Med Chem. 2015 Aug 26:1-6. [Epub ahead of print]

Prohic A, Jovovic Sadikovic T, Krupalija-Fazlic M, Kuskunovic-Vlahovljak S. Malassezia species in healthy skin and in dermatological conditions. Int J Dermatol. 2016 May;55(5):494-504. doi: 10.1111/ ijd.13116. Epub 2015 Dec 29.

Rajič B, Arapović J, Raguž K, Bošković M, Babić SM, Maslać S. Eradication of Blastocystis hominis prevents the development of symptomatic Hashimoto's thyroiditis: a case report. J Infect Dev Ctries. 2015 Jul 30;9(7):788-91. doi: 10.3855/ jidc. 4851 .

Rašić S, Rebić D, Hasić S, Rašić I, Delić Šarac M. Influence of Malondialdehyde and Matrix Metalloproteinase-9 on Progression of Carotid Atherosclerosis in Chronic Renal Disease with Cardiometabolic Syndrome. Mediators Inflamm. 2015;2015:614357. doi: 10.1155/2015/614357. Epub 2015 Oct 11 .

Rebić D, Matovinović MS, Rašić S, Kes P, HamzićMehmedbašić A. The Effect of Preserved Residual Renal Function on Left Ventricular Structure in NonAnuric Peritoneal Dialysis Patients. Kidney Blood Press Res. 2015;40(5):500-8. doi: 10.1159/000368526. Epub 2015 Oct 4.
Rebić D, Rašić S, Hamzić-Mehmedbašić A, Džemidžić J, Kurtalić E. Valvular calcification and left ventricular modifying in peritoneal dialysis patients. Ren Fail. 2015;37(8):1316-22. doi: 10.3109/0886022X.2015.1073495. Epub 2015 Aug 14.

Salkic NN, Cickusic E, Jovanovic P, Denjagic MB, Iljazovic-Topcic S, Bevanda M, Ahmetagic S. Online combination algorithm for non-invasive assessment of chronic hepatitis B related liver fibrosis and cirrhosis in resource-limited settings. Eur J Intern Med. 2015 Oct;26(8):628-34. doi: 10.1016/j. ejim.2015.07.005. Epub 2015 Jul 18.

Santrac V, Nedic DN, Maric J, Nikolic S, Stevanovic O, Vasilev S, Cvetkovic J, Sofronic-Milosavljevic L. The first report of Trichinella pseudospiralis presence in domestic swine and T. britovi in wild boar in Bosnia and Herzegovina. Acta Parasitol. 2015 Sep;60(3):471-5. doi: 10.1515/ap-2015-0066.

Sartelli M, Abu-Zidan FM, Catena F, Griffiths EA, Di Saverio S, Coimbra R, Ordoñez CA, Leppaniemi A, Fraga GP, Coccolini F, Agresta F, Abbas A, Abdel Kader S, Agboola J, Amhed A, Ajibade A, Akkucuk S, Alharthi B, Anyfantakis D, Augustin G, Baiocchi G, Bala M, Baraket O, Bayrak S, Bellanova $\mathrm{G}$, Beltràn $\mathrm{MA}$, Bini R, Boal $\mathrm{M}$, Borodach $\mathrm{AV}$, Bouliaris K, Branger F, Brunelli D, Catani M, Che Jusoh A, Chichom-Mefire A, Cocorullo G, Colak E, Costa D, Costa S, Cui Y, Curca GL, Curry T, Das K, Delibegovic S, Demetrashvili Z, Di Carlo I, Drozdova N, El Zalabany T, Enani MA, Faro M, Gachabayov M, Giménez Maurel T, Gkiokas G, Gomes CA, Gonsaga RA, Guercioni G, Guner A, Gupta S, Gutierrez S, Hutan M, Ioannidis O, Isik A, Izawa Y, Jain SA, Jokubauskas M, Karamarkovic A, Kauhanen S, Kaushik R, Kenig J, Khokha V, Kim JI, Kong V, Koshy R, Krasniqi A, Kshirsagar A, Kuliesius Z, Lasithiotakis K, Leão P, Lee JG, Leon M, Lizarazu Pérez A, Lohsiriwat V, López-Tomassetti Fernandez E, Lostoridis E, Mn R, Major P, Marinis A, Marrelli D, Martinez-Perez A, Marwah S, McFarlane M, Melo RB, Mesina C, Michalopoulos N, Moldovanu R, Mouaqit O, Munyika A, Negoi I, Nikolopoulos I, Nita GE, Olaoye I, Omari A, Ossa PR, Ozkan Z, Padmakumar R, Pata F, Pereira Junior GA, Pereira J, Pintar T, Pouggouras K, Prabhu V, Rausei S, Rems M, Rios-Cruz D, Sakakushev B, Sánchez de Molina ML, Seretis C, Shelat V, Simões RL, Sinibaldi G, Skrovina M, Smirnov D, Spyropoulos C, Tepp J, Tezcaner T, Tolonen M, Torba M, Ulrych J, Uzunoglu MY, van Dellen D, van Ramshorst GH, Vasquez G, Venara A, Vereczkei A, Vettoretto N, Vlad N, Yadav SK, Yilmaz TU, Yuan KC, Zachariah SK, Zida M, Zilinskas J, Ansaloni L. Global validation of the WSES Sepsis Severity Score for patients with complicated intra- 
abdominal infections: a prospective multicentre study (WISS Study). World J Emerg Surg. 2015 Dec 16;10:61. doi: 10.1186/s13017-015-0055-0. eCollection 2015.

Stankovic MS, Turuntas V, De Luka SR, Jankovic S, Stefanovic S, Puskas N, Zaletel I, MilutinovićSmiljanic S, Trbovich AM. Effects of Il-33/St2 pathway on alteration of iron and hematological parameters in acute inflammation. Exp Mol Pathol. 2015 Dec;99(3):687-92. doi: 10.1016/j. yexmp.2015.11.016. Epub 2015 Nov 11.

Šabanagić-Hajrić S, Suljić E, Kučukalić A. Fatigue during multiple sclerosis relapse and its relationship to depression and neurological disability. Psychiatr Danub. 2015 Dec;27(4):406-12.

Šutalo N, Šoljić M, Kozomara D, Petričević J, Glibo DB, Bošnjak A, Čuljak V. The influence of dreinage in the treatment of chronic pilonidal sinus disease using midline closure. Psychiatr Danub. 2015 Dec;27 Suppl 2:593-5.

Veličković V, Jović M, Nalić E, Višnjić A, Radulović O, Šagrić Č, Ćirić M. Knowledge, Attitudes Toward, and Acceptability of Genetic Modification among Western Balkan University Students of Life Sciences (AGREE Study). J Am Coll Nutr. 2016 Feb-Mar;35(2):150-62. doi: 10.1080/07315724.2014.1003115. Epub 2015 Jun 1.

Vodenčarević AN, Terzić S, Terzić A. Endophthalmitis After Intravitreal Injections: Incidence, Presentation, Management, and Visual Outcome. Am J Ophthalmol. 2015 Oct;160(4):843-4. doi: 10.1016/j.ajo.2015.07.003. Epub 2015 Jul 30.

Weltermann BM, Gerasimovska-Kitanovska B, Thielmann A, Chambe J, Lingner H, Pirrotta E, Buczkowski K, Tekiner S, Czachowski S, Edirne T, Zielinski A, Yikilkan H, Koskela T, Petrazzuoli F, Hoffman RD, Petek Šter M, Guede Fernández C, Uludağ A, Hoffmann K, Mevsim V, Kreitmayer
Pestic S. Self-Care Practices for Common Colds by Primary Care Patients: Study Protocol of a European Multicenter Survey-The COCO Study. Evid Based Complement Alternat Med. 2015;2015:272189. doi: 10.1155/2015/272189. Epub 2015 Sep 2.

Yahia ME, Tolba RE, El-Bedwehy NA, El-Labany SK, Moslem WM. Rogue waves lead to the instability in GaN semiconductors. Sci Rep. 2015 Jul 24;5:12245. doi: 10.1038/srep12245.

Zerem E, Imširović B, Loga-Zec S, Kunosić S, Hujdurović A, Zerem O. Treatment of Recurrent Pancreatic Pseudocysts with Proven Communication between Pseudocyst and Pancreatic Duct by Longterm Percutaneous Drainage. Ann Acad Med Singapore. 2015 Nov;44(11):542-4.

Zerem E, Lincender-Cvijetić L, Kurtčehajić A, Samardžić J, Zerem O. Symptomatic Gallbladder Sludge and its Relationship to Subsequent Biliary Events. J Clin Gastroenterol. 2015 Oct;49(9):795-6. doi: 10.1097/MCG.0000000000000361.

Zerem E, Omerović M, Zerem O. What is the optimal treatment for hepatic abscess and infected biloma? Gastrointest Endosc. 2015 Nov;82(5):971-2. doi: 10.1016/j.gie.2015.06.011.

Zielinski C, Lang I, Beslija S, Kahan Z, Inbar MJ, Stemmer SM, Anghel R, Vrbanec D, Messinger D, Brodowicz T. Predictive role of hand-foot syndrome in patients receiving first-line capecitabine plus bevacizumab for HER2-negative metastatic breast cancer. Br J Cancer. 2016 Jan 19;114(2):163-70. doi: 10.1038/bjc.2015.419. Epub 2015 Dec 10.

Zvorničanin J, Zvorničanin E. The Diagnostic Accuracy of Bedside Ocular Ultrasonography for the Diagnosis of Retinal Detachment: A Systematic Review and Meta-analysis. Ann Emerg Med. 2015 Sep;66(3):342-3. doi: 10.1016/j. annemergmed.2015.05.011. 